\title{
Synchronization of two different fractional order chaotic systems with unknown parameters using a robust adaptive nonlinear controller
}

\begin{abstract}
In this paper, a robust adaptive nonlinear feedback controller scheme is proposed to realize the synchronization between two different fractional-order chaotic systems with fully unknown parameters, external disturbance and uncertainties. Bounds of the uncertainties and external disturbance assumed to be unknown. A new theorem is presented to satisfy Lyapunov stability condition in fractional-order systems when their parameters are fully unknown with external disturbance and uncertainties. Numerical simulations are applied using MATLAB software to show the effectiveness of the proposed schemes.
\end{abstract}

Keyword: Synchronization; Adaptive; Chaos; Fractional; Control; Stability; Lyapunov 